\title{
Family participation in treatment, post-discharge appointment and medication adherence at a Nigerian psychiatric hospital
}

Ademola B. Adeponle, Brett D. Thombs, Moruf L. Adelekan and Laurence J. Kirmayer

\begin{abstract}
Summary
In low-income countries, clinicians must seek strategies to improve treatment adherence that are non-resource intensive and easily integrated into existing treatment structures. We conducted a prospective observational cohort study to investigate the relationship of family engagement in treatment during hospitalisation with post-discharge appointment and medication adherence in 81 patients from a Nigerian psychiatric hospital. After controlling for gender,
\end{abstract}

diagnosis, mental state at discharge, and marital status, family involvement was significantly associated with appointment $(P=0.047)$ but not medication adherence $(P=0.590)$. Studies are needed to determine whether interventions based on engaging families in treatment can improve post-discharge adherence in this setting.

Declaration of interest None.
Non-adherence to psychiatric treatment undermines treatment effectiveness, increases risk of relapse and predisposes to poorer outcomes. ${ }^{1,2}$ A systematic review found that one of four patients with psychosis do not keep scheduled post-discharge appointments, and almost one in three do not take medications as prescribed. Most studies reviewed, however, were from Europe or the USA. ${ }^{2}$ In Europe and the USA, psychoeducation programmes, family therapy and community-based interventions have been used successfully to improve adherence. ${ }^{2-5}$ Although not all studies have reported significant effects on adherence, most interventions that have improved adherence have also reported positive clinical outcomes, such as fewer rehospitalisations. ${ }^{1}$ One study from Africa examined post-discharge treatment adherence among 387 patients at a Nigerian psychiatric unit and found that $46 \%$ had defaulted by 3 months. ${ }^{6}$ In low-income countries such as Nigeria, structural barriers, including poverty, poor infrastructure and the absence of formal social welfare services and trained staff, limit the applicability of adherence strategies used in high-income countries. ${ }^{5}$ In this context, clinicians must seek pragmatic approaches more feasibly integrated into existing treatment structures. One such strategy involves engaging the families in treatment programmes in order to facilitate post-discharge support that, in high-income countries, might typically be provided by community workers. It is not known, however, whether participation by family members in in-patient care in low-income countries predicts better post-discharge adherence. The objective of this study was to investigate whether family involvement in treatment during hospitalisation is independently associated with greater post-discharge appointment and medication adherence.

\section{Method}

This was a prospective study of patients admitted for in-patient care to the Federal Psychiatric Hospital, Kaduna, Nigeria. The hospital is a 100-bed public regional psychiatric hospital that primarily receives patients from north-west and north-central Nigeria and the federal capital territory, Abuja. Kaduna is a cosmopolitan city of 1.2 million people and is regarded as the capital city of northern Nigeria. Patients admitted between July and December 2004 and taking their medications as prescribed at discharge were eligible for the study. A study physician assessed patients' mental state, family involvement in treatment and medication side-effects prior to discharge. The Brief Psychiatric Rating Scale ${ }^{7}$ (BPRS), which has been used previously in Nigeria, ${ }^{8}$ was used to assess mental state. Medication side-effects were assessed via direct observation and review of case notes. Family involvement was defined using two criteria: (a) the patient's family must have visited during admission (at least once every 2 weeks), and (b) they must have attended at least three treatment sessions (an intake interview session within 2 weeks of admission, a second when the patient had become clinically stable, and a third in the week of discharge). At 3-month follow-up, treatment adherence was recorded and medication adherence assessed. Non-adherers to treatment were defined as patients who did not attend scheduled appointments and who did not re-schedule within 2 weeks. Patients who did not attend appointments were followed up at home by a female social worker and male physician in order to assess medication adherence, which was assessed through interviews with patients and family members. Non-adherence was defined as the failure to take prescribed medications for a week or longer, or using medications in less-than-prescribed doses. Ethical approval for the study was given by the hospital's research and ethics committee, and informed consent was obtained from all patients prior to discharge.

Data analyses included comparisons of demographic and clinical variables between adherers and non-adherers to appointments and medications using chi-squared tests for categorical variables and $t$-tests for continuous variables. Multivariable logistic regression was used to test whether family involvement in treatment prior to discharge was associated with non-adherence to post-discharge appointments and medication, controlling for variables that were related to appointment or medication adherence on a bivariate basis $(P<0.10)$.

\section{Results}

There were 81 patients who met study criteria and all consented to take part in the study. Slightly more than half were male $(54.3 \%$, $n=44)$. Most patients $(67.9 \%, n=55)$ were $21-40$ years old; $30.9 \%$ $(n=25)$ were married; $64.2 \%(n=52)$ had $\geqslant 10$ years of education; $53.1 \%(n=43)$ were employed or in school; and $44.4 \% \quad(n=36)$ lived $\geqslant 20 \mathrm{~km}$ from the hospital. Diagnoses included non-affective psychosis $(59.3 \%, n=48)$, affective disorders $(24.7 \%, n=20)$, and substance-related disorders $(16.0 \%, n=13)$. Illness duration was 
$\geqslant 1$ year for $69.1 \%(n=56)$ of patients, and $71.6 \%(n=58)$ had received previous treatment. Prescribed medications included traditional antipsychotics $(92.6 \%, n=75)$, anticholinergics (95.1\%, $n=76)$, mood stabilisers (18.5\%, $n=15)$, antidepressants $(16.0 \%, n=13)$ and depot antipsychotics $(38.3 \%, n=31)$. The families of $75.3 \%(n=61)$ of patients were involved in treatment.

At 3 months post-discharge, $41(50.6 \%)$ patients were adherent with appointments. Of the 40 who were non-adherent, 13 were not located for 3-month follow-up owing to incorrect/ untraceable addresses or relocation. Thus, 68 patients were assessed, of whom $42.6 \% \quad(n=29)$ were adherent. As shown in online Table DS1, adherers to appointments were significantly less likely to have a substance misuse disorder and had lower BPRS scores at discharge. Their families were more often involved in treatment. Adherers to medication were significantly less likely to have a substance misuse disorder. After controlling for gender, diagnosis, BPRS score at discharge and marital status, patients whose families were involved in treatment continued to be significantly more likely to adhere to scheduled appointments (odds ratio $(\mathrm{OR})=3.66,95 \%$ confidence interval (CI) 1.02-13.16, $P=0.047$ ), and somewhat more likely to adhere to prescribed medication, albeit not significantly $(\mathrm{OR}=1.51,95 \%$ CI $0.34-$ $6.70, P=0.590)$. Similar results were obtained when the 13 nonlocated patients were treated as non-adherent to medication $(\mathrm{OR}=2.13,95 \%$ CI $0.56-8.20, P=0.270)$. Results did not change for treatment or medication adherence if only patients $\geqslant 21$ years old were included.

\section{Discussion}

The main finding of this study was that involvement of families in treatment during psychiatric hospitalisation at a Nigerian hospital predicted significantly improved post-discharge adherence to appointments, but not to medication. The non-significant finding for medication adherence may have been due to the moderate sample size of the study, although it should also be noted that accurate measurement of medication adherence is methodologically challenging. Interview and case-note methods were used to assess medication adherence and side-effects, but may have been less accurate than more objective measures such as pill counts, blood assays or standardised side-effects rating scales. ${ }^{9}$ This was an observational study, so it is not known whether these findings would translate into an effective intervention programme. Patients with family involvement may have differed from patients without family involvement in ways related to adherence that were not controlled for in this study. We did not assess factors that may have influenced family involvement such as the non-existence of living family members, alienation from families or stigmatisation. With $92 \%$ of patients prescribed antipsychotic medications, the question might be posed as to whether there was a problem of antipsychotic over-prescription and whether this might explain why some patients stopped their medications, given that about $40 \%$ of patients had diagnoses of substance-related or affective disorders. The explanation for the apparently high antipsychotic use lies in the fact that virtually all admitted patients, including those diagnosed with substancerelated and affective disorders, presented with psychotic symptoms, a common occurrence in Nigerian psychiatric hospital settings. ${ }^{10}$

This study suggests that engaging the family during treatment may improve post-discharge adherence. Although it is generally acknowledged that the family remains an important resource for the support and care of psychiatric patients in most African countries, existing mental health policies do not emphasise this resource. ${ }^{11}$ In line with international trends, mental healthcare in poorer countries is increasingly being integrated into primary care. ${ }^{5}$ In Nigeria, efforts over the past decade to develop a nationwide primary healthcare-based community psychiatry programme have generally not been effective. There is precedent, however, for engaging family members in psychiatric treatment in Nigeria. One of the earliest successful models of community psychiatry care, the Aro village system, was started in the country in the 1950s. It was a widely acclaimed, innovative programme that sought to engage family members in treatment programmes for hospitalised psychiatric patients, and which had reliance on family ties and provision of a culturally salient service as its twin bedrocks. ${ }^{12}$ Whether or not involving families in in-patient psychiatric care improves post-discharge adherence in settings such as Nigeria, however, needs to be demonstrated in a study designed specifically for that purpose.

Ademola B. Adeponle MD, Federal Neuro-Psychiatric Hospital, Kaduna, Nigeria; Brett D. Thombs, PhD, Department of Psychiatry, McGill University and SMBD-Jewish General Hospital Montreal, Quebec, Canada; Moruf L. Adelekan, MD MRCPsych, Royal Blackburn Hospital, Blackburn, UK; Laurence J. Kirmayer, MD, FRCCP, Division of Social and Transcultural Psychiatry, Department of Psychiatry, McGill University and SMBD-Jewish General Hospital Montreal, Quebec, Canada

Correspondence: Ademola B. Adeponle, Dision of Social and Transcultural Psychiatry, Department of Psychiatry, 1033 Pine Avenue West, Montreal, Quebec, H3A 1A1, Canada. Email:dradeponleab@yahoo.com

First received 6 Mar 2008, final revision 2 Jun 2008, accepted 9 Jul 2008

\section{References}

1 Zygmunt A, Olfson M, Boyer CA, Mechanic D. Interventions to improve medication adherence in schizophrenia. Am J Psychiatry 2002; 159: 1653-64.

2 Nosé M, Barbui C, Gray R, Tansella M. Clinical interventions for treatment non-adherence in psychosis: meta-analysis. Br J Psychiatry 2003; 183 : 197-206.

3 M Nosé, C Barbui, M Tansella. How often do patients with psychosis fail to adhere to treatment programmes? A systematic review. Psychol Med 2003; 33: 1149-60.

4 Saxena S, Paraje G, Sharan P, Karam G, Sadana R. The 10/90 divide in mental health research: trends over a 10-year period. Br J Psychiatry 2006; 188 $81-2$.

5 Breen A, Swartz L, Joska J, Flisher AJ, Corrigall J. Adherence to treatment in poorer countries. A New Research Direction? Psych Services 2007; 58: 567-8.

6 Ikwuagwu PU, Nafziger JC, Ihezue UH, Ohaeri JE. A study of the social and clinical characteristics of in-patients at a psychiatric unit in northern Nigeria. West Afr J Med 1994; 13: 191-5.

7 Overall JE, Gorham DR. The Brief Psychiatric Rating Scale (BPRS). Psychological Reports 1962; 10: 799-812.

8 Ohaeri JU, Otote DI. Subtypes and factors of schizophrenia in an acutely ill Nigerian sample. Psychopathology 2003; 36: 181-9.

9 Day JC, Wood G, Dewey M, Bentall RP. A self-rating scale for measuring neuroleptic side-effects. Validation in a group of schizophrenic patients. Br J Psychiatry 1995; 166: 650-3.

10 Makanjuola RO. Recurrent unipolar manic disorder in the Yoruba Nigerian: further evidence. Br J Psychiatry 1985; 147: 434-7.

11 Gureje O, Alem A. Mental health policy development in Africa. Bull World Health Organ 2000; 78: 475-82.

12 Sadowsky J. Imperial Bedlam: Institutions of Madness in Colonial Southwest Nigeria. University of California Press, 1999. 